\title{
Assessment of Various Low-Profile Mechanical Vortex Generators in Controlling a Shock-Induced Separation
}

\author{
S. B. Verma* and C. Manisankari \\ National Aerospace Laboratories, Bangalore 560 017, India
}

DOI: $\underline{10.2514 / 1 . J 055446}$

\begin{abstract}
An experimental investigation was conducted to assess the effectiveness of five microvortex generator configurations in controlling an incident shock-induced separation associated with a 14 deg shock generator in a Mach 2.05 flow. The vortex generator configurations studied include the Ashill, Anderson, split-Anderson, trapezoidal, and ramp-vane designs. Each device height $h$ spanned $30 \%$ of the local boundary-layer thickness $\delta$ estimated just upstream of the separation for no control. An array of each control device configuration was implemented $10 \delta$ upstream of the separation location for no control. Additionally, one ramp-vane device with $h / \delta=0.5$ was also tested. Out of all the configurations tested, the ramp-vane device $(h / \delta=0.5)$ shows the maximum downstream shift $(21 \%)$ in separation location. This device and the split-Anderson configuration $(h / \delta=0.3)$ both show a reduction in the maximum rms values by 26 and $24 \%$, respectively. The study on ramp-vane devices $(h / \delta=0.3,0.5)$ further shows that the size of the split relative to the device height also seems to be an important parameter. For the ramp-vane devices $(h / \delta=0.3,0.5)$, a smaller intervane spacing of $1.7 h(h / \delta=0.5)$ instead of $3 h$ $(h / \delta=0.3)$ shows a very effective control. From this perspective, providing a split size of $1 h$ in split-Anderson device has also shown favorable results.
\end{abstract}

\section{Introduction}

$\mathbf{T}$ HE process of flow deceleration in a supersonic intake occurs through a complex system of shocks. The challenge lies in minimizing total pressure losses across the shocks over a finite length and providing uniform flow conditions at the engine face. However, the interaction of these shocks with the boundary layer growing on the intake walls is inevitable. This increases the likelihood of shockinduced separation, which can alter the process of deceleration and introduce flow distortions that can degrade/limit the inlet performance and, in the worst case, lead to engine unstart. Also the shock unsteadiness [1, 2] associated with separated interactions can contribute toward structural loads in supersonic inlets [3] and is of concern. As a result, it becomes imperative to improve the health of the incoming boundary layer so as to control the severity of the shockinduced boundary-layer separation.

Boundary-layer manipulation [4,5] techniques have been used in the past to replace the low-momentum fluid in the incoming boundary layer with the high-momentum fluid of the main flow so that the reenergized boundary layer is able to sustain relatively higher adverse pressure gradients to avoid or delay separation. The most commonly used control in this category uses surface suction or bleed ahead of the shock-induced interaction. This method is not only found to be effective in preventing or delaying shock-induced separation [6-8] but also makes the resulting boundary-layer thinner reducing flow distortions. As a result, this control technique has found several hardware applications. However, the system associated with them is quite complex and expensive [9]. Furthermore, the boundary-layer mass flow removed through suction also reduces the mass flow rate reaching the engine that needs to be compensated by increasing the inlet area. This leads to weight and performance penalties and increases ram air drag [10]. As a result, it is desirable to explore innovative control methods that can reduce or perhaps serve

Received 27 June 2016; revision received 16 January 2017; accepted for publication 28 February 2017; published online 12 May 2017. Copyright (C) 2017 by the authors. Published by the American Institute of Aeronautics and Astronautics, Inc., with permission. All requests for copying and permission to reprint should be submitted to CCC at www.copyright.com; employ the ISSN 0001-1452 (print) or 1533-385X (online) to initiate your request. See also AIAA Rights and Permissions www.aiaa.org/randp.

*Deputy Head, Experimental Aerodynamics Division, Council of Scientific and Industrial Research; sbverma@nal.res.in. Associate Fellow AIAA.

${ }^{\dagger}$ Scientist, Experimental Aerodynamics Division, Council of Scientific and Industrial Research; mani@nal.res.in. as an alternative to the conventional bleed while maintaining the intake performance [10].

Addition of momentum to the near-wall flow region to manipulate the boundary-layer profile has seen several innovative ways to derive energy from the freestream. These are primarily based on developing control techniques that help to generate vortices close to the wall so as to initiate the process of transfer of the higher momentum of the outer flow near to the wall. These can be broadly classified into those using an active or passive approach. Although the former approach uses plasma jets or steady/pulsed-air jets (normal or angled to the main flow), which on interaction with the oncoming flow generate streamwise vortices, the latter approach uses an array of vanes in coor counter-rotating configuration, delta ramps, etc., to initiate the same effect. Each of the preceding methods of flow control has their advantages and disadvantages. For example, the active vortex generators (VGs) $[11,12]$ score over the mechanical VGs in that they can be turned on or off on demand, eliminating any device or parasitic drag. However, these devices require input of extra energy and may require higher installation and maintenance costs. The mechanical VGs, on the other hand, induce device drag, which can slightly offset their benefits [13]. However, this is more relevant to conventional vane-type VGs with height of the order of boundary-layer thickness $(h / \delta \geq 1)$. As a result, micro-VGs (MVGs) with $h / \delta<1$ are preferable for energizing the turbulent boundary layer while minimizing the device drag [13-15]. The challenge, however, lies in introducing similar control effects as the conventional VGs [13]. Various mechanical VG configurations such as the microramps $[\underline{10}, \underline{16}-\underline{20}]$, split ramps [21], and ramp vane-type designs [10,22-26] have been studied in literature. Computational studies on flow development in the wake of the micro-VG devices and their effect on the shock-induced interactions have also been conducted [22,27-30]. All these investigations, both experimental and computational, support the effectiveness of these devices in controlling shockinduced interactions. The device height with respect to the boundarylayer thickness has also been found to be an important parameter in controlling the separation $[10,20]$. Studies reveal that the general flow features [10] and the momentum flux added to near-wall region $[20,27]$ scale linearly with device height. As a result, a larger-sized microramp was found to be more effective in stabilizing the interaction. A detailed study by Anderson et al. [18] further reports that most microramp configurations introduce similar separation control and that these control devices provide a more fail-safe option (being mechanically rugged) associated with their designs over the conventional vane-type VGs. Similar suggestions have been indicated by others [15] as well. Verma and Hadjadj [31] give a 
detailed comprehensive view of the various flow control techniques used in shock-wave/boundary-layer interactions including those such as the shock control bumps (SCBs) and plasma jets. In case of $\mathrm{SCBs}$, because the design is based on the generation of weak compression waves ahead of the shock, this device has been reported to have a strong potential for transonic wings [32]. On the other hand, plasma jets assist in locking the pulsing energy of the jets to that of the shock front, which helps to reduce the magnitude of shock unsteadiness [33]. But because only $10 \%$ of the plasma energy is available for jets, this control needs to be placed about $1.5 \delta$ upstream of separation. A few studies also offer explanations toward the possible source of shock unsteadiness in such interactions such as those arising from the coupling mechanism between the separation bubble and the shock [34], the interaction strength [35], and upstream effects [36].

A few computational [22,27] and experimental [24,25] efforts have also been made to compare the effectiveness of various microVG configurations but primarily in the transonic flow regime. However, to the best of the authors' knowledge, not much work has been reported that compares and evaluates the effectiveness of various micro-VG devices in a purely supersonic interaction $[10,18,20]$. The control of supersonic SBLIs reported by Babinsky et al. [10] and Giepman et al. [20] have only used microramps of Anderson configuration with height variation. The only study that does report the use of various micro-VG devices in a Mach 2 incident shock-induced separation is that of Anderson et al. [18]. The study, however, does not indicate the impact of each VG design on the extent of separation or on the separation shock unsteadiness. The present experimental study therefore aims to assess the effectiveness of various such micro-VG devices in controlling an incident shockinduced separation associated with a $14 \mathrm{deg}$ shock generator (SG) in a Mach 2.05 flow. For this purpose, the performances of the conventionally studied microramp configurations, namely the Ashill et al. [17], the Anderson et al. [18], and the split-Anderson [21] types, are compared with those of trapezoidal (TRZ) and the ramp-vane (RV1, based on the ramp-vane design proposed by Babinsky et al. [10]) configurations in controlling the extent of separation. All these control configurations had a device height that spanned $30 \%$ of the local boundary-layer thickness $(0.3 \delta)$ estimated just upstream of the separation for no-control case. Additionally, one case of ramp-vane (RV2) with height of $0.5 \delta$ is also studied. The overall objective is to assess the effectiveness of various mechanical vortex generator configurations in controlling the extent of separation or its associated shock-foot unsteadiness Detailed investigation of the interaction is made using color spark schlieren (500 ns), surface oil visualization, mean pressure measurements using electronic pressure scanners, and real-time pressure measurements using fast piezoresistive Kulite pressure sensors.

\section{Experimental Setup and Procedure}

\section{A. Wind-Tunnel Facility and Model Details}

Tests were conducted in the $0.46 \times 0.3 \mathrm{~m}$ blowdown trisonic wind-tunnel at National Aerospace Laboratories. The test Mach number was $2.05 \pm 0.02\left(U_{\infty}=523 \mathrm{~m} \cdot \mathrm{s}^{-1}\right)$, whereas the stagnation pressure $P_{0}$ and temperature $T_{0}$ were $208.5 \mathrm{kPa} \pm 2 \%$ (absolute) and $298 \mathrm{~K} \pm 0.4 \%$, respectively. The unit Reynolds number $R e / L$ of the flow was $25.257 \times 10^{6} \mathrm{~m}^{-1}$. The wall temperature was assumed adiabatic, and the turbulence levels in the tunnel were approximately $0.2 \%\left(\% C_{p, r m s}\right)$. The flat-plate model was mounted on a sting along the tunnel centerline as shown in Fig. 1a. An incident shock wave $(\beta=42.93 \mathrm{deg})$, generated using a fixed wedge (of $220 \mathrm{~mm}$ width) with a flow turning angle of $14 \mathrm{deg}$, is made to impinge on a plat plate (Fig. 1b). The Mach number downstream of the incident shock is 1.53 . The flat plate of the model was $34 \mathrm{~cm}$ long with a span of $11 \mathrm{~cm}$ (Fig. 1c). The Reynolds number $R e_{x}$ based on the entire flat-plate length was $8.58 \times 10^{6}$. For mean pressure measurements, 22 port locations ( $\mathrm{P} 1$ to $\mathrm{P} 22$ ) are available along the centerline, whereas for unsteady pressure study, the transducers are available only for the first 12 locations marked K1 to K12 and were located $5 \mathrm{~mm}$ off the centerline, as shown in Fig. 1c. As a consequence of the latter, rms values are available only for these locations (results discussed in Sec. III). The test section was equipped with two optical windows of $280 \mathrm{~mm}$ diameter to provide optical access to the interaction region. No side fences were used on the plate to facilitate schlieren imaging. A boundary-layer trip, made of 60 grit

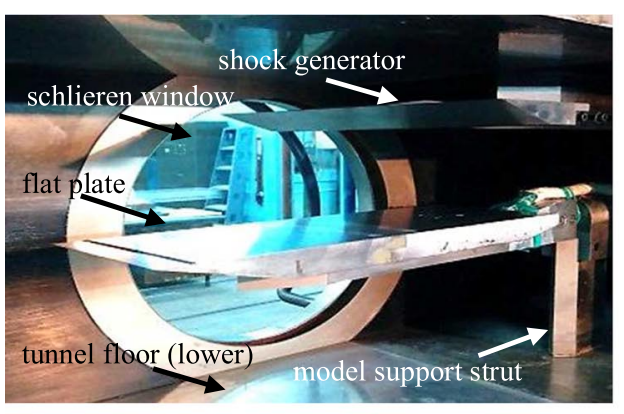

a)

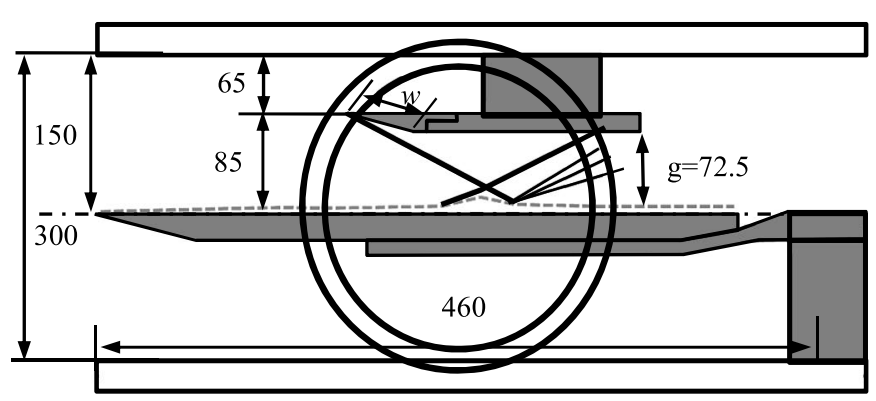

b)

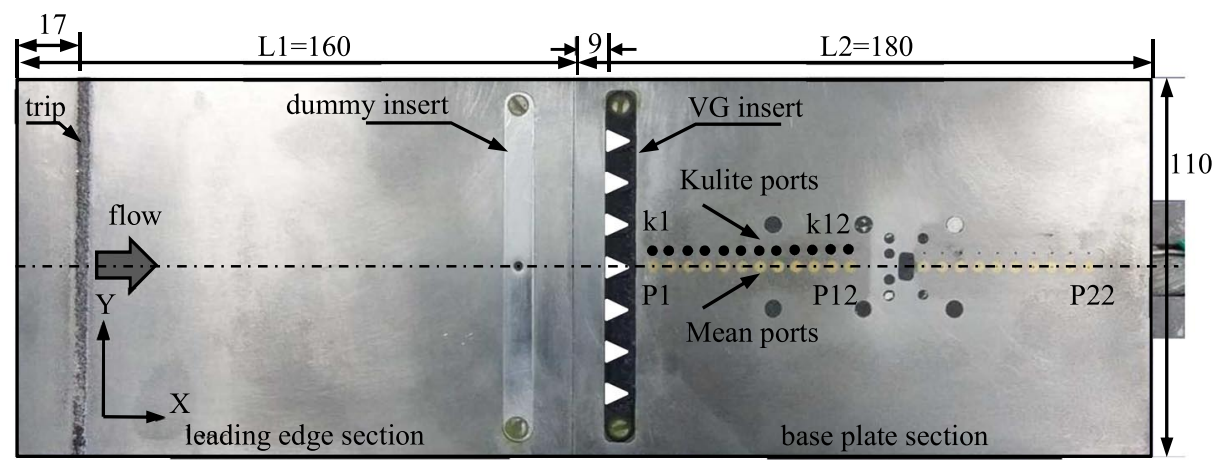

c)

Fig. 1 Details of a) the model mounted in the wind-tunnel, b) schematic of the experimental set-up, and c) model details with pressure sensors and VG location. All dimensions are in millimeters. 


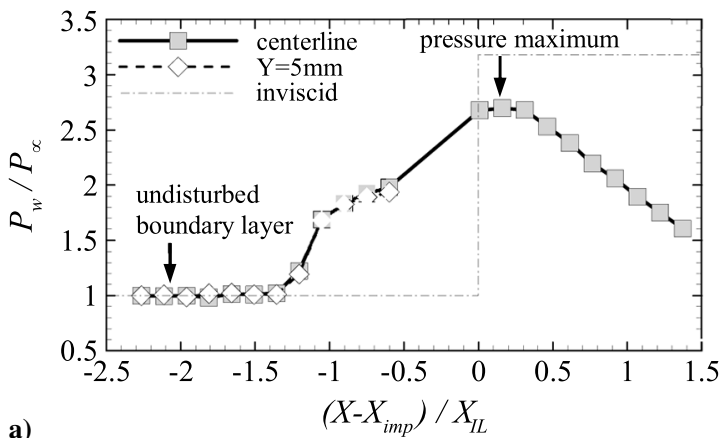

a)

$$
\left(X-X_{\text {imp }}\right) / X_{I L}
$$

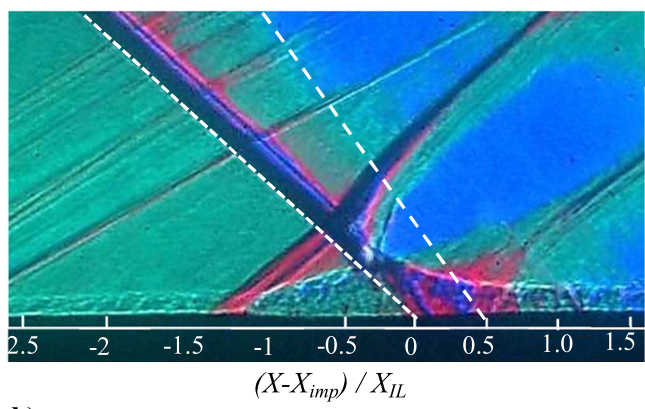

b)

Fig. 2 Representations of a) the streamwise mean pressure distribution for no control, and b) schlieren image of the corresponding flow.

carborundum particles spanning $4 \mathrm{~mm}$ in length and placed at $17 \mathrm{~mm}$ from the leading edge, was used to ensure a turbulent boundary layer. For the present model and the test arrangement, the $g / w$ value is 1.4 (Fig. 1b). This made sure that the expansion fan emanating from the downstream end of the wedge did not interact with the incident shock and minimized the effect on the interaction generated by it (Fig. 1b). Using inviscid calculations, the distance of the first characteristic of expansion fan (long dashed line) and the incident shock impingement location (short dashed line, Fig. 2b) was found to be approximately $4 \delta$ and is seen in the wall pressure distribution as a pressure maximum (Fig. 2a) and is the result of the compression waves at reattachment interacting with the expansion fan coming from the shoulder of the SG (Fig. 2b). The inviscid pressure ratio for this interaction is 3.2. The interaction length $X_{I L}$, defined as distance between extrapolated wall impact points of incident $\left(X_{\text {imp }}\right)$ and separation/reflected shocks $\left(X_{s}\right)$ as evaluated from the schlieren image, and the separation length $X_{\mathrm{SL}}$ (the distance between the first rise in wall pressure along the centerline and $X_{\text {imp }}$ ) are $33 \pm 1$ and $45 \pm 1 \mathrm{~mm}$, respectively. The difference between $X_{I L}$ and $X_{\mathrm{SL}}$ is the region of intermittent separation. In this region, the wall pressure begins to first sense the rise in pressure due to back-and-forth shock motions and ends where the flow physically separates from the wall. Thus, although the schlieren images and surface oil visualization help to reveal $X_{I L}$, the real-time pressure measurements using Kulite transducers help to pick the intermittent signals ahead of physical separation and hence assist in detecting $X_{\mathrm{SL}}$. It can also be seen in Fig. 2a, from the Kulite and ESP pressure distributions, that the surface pressure rise across the interaction is very uniform on either side of the centerline. Further, the separation line (shown in the surface oil picture for no control shown in Sec. III.C) is seen to be nearly straight for almost $20 \mathrm{~mm}$ on either side of the model centerline. However, because of the finite spanwise extent of the flat plate, three-dimensional effects begin to influence the interaction toward the outer edges of the flat plate. It may therefore be borne in mind that the discussions of the results in this paper are limited only to that region of the flow about the centerline up to which a straight separation line is seen for no control. The experimental conditions and the undisturbed boundarylayer properties are shown in Table 1 . Here, $S_{e}^{*}$ and $L^{*}$ correspond to the separation criteria and the nondimensional interaction length (value obtained from the best fit line) based on scaling described by Souverein et al. [35]. The mean skin friction coefficient $c_{f}$, after correction for turbulent flows [37], is $2.1 \times 10^{-3}$.

Control devices of various configurations were tested to compare their capability to control the flow interaction. Each of these devices was arranged in the form of an array of single-row micromechanical VGs. Care was taken, however, to ensure that a VG device of each configuration was always placed on the model centerline and in line with the streamwise row of the mean pressure measurement locations $\mathrm{P} 1$ to P22. Figure 3 shows the schematic of the various $\mathrm{VG}$ configurations used in the present study. The five microramp VG configurations studied in the present work are 1) Ashill et al. type [17], 2) Anderson et al. [18], 3) split-Anderson type [21], 4) trapezoidal (TRZ), and 5) ramp-vane (RV1) based on the rampvane design proposed by $[10,27]$. However, in the present design, the vertex of each RV1 has a finite thickness (of $0.5 \mathrm{~mm}$, Fig. 3e) and is not sharp, as studied earlier [25]. The angle of incidence $\alpha$ for all the VGs is $24 \mathrm{deg}$ (Fig. 3), except for the Ashill type, for which $\alpha=14 \mathrm{deg}$. All these VG configurations have a similar height $h=1 \mathrm{~mm}$ or $h=0.3 \delta$ and an interdevice spacing (center-to-center) of $s=12 h$, resulting in an array of seven control devices of each configuration. Additionally, a ramp-vane (RV2) device with height $0.5 \delta$ was also studied. The configuration RV2 is similar to the RV1 (Fig. 3e), except that the height of the downstream end of the ramp is increased to $1.75 \mathrm{~mm}(0.5 \delta)$, whereas all other dimensions remained same as for $h=1 \mathrm{~mm}$ case as shown in Fig. 3f. This was done intentionally so that, when the height of RV1 is increased, seven control devices of RV2 configuration still fit well within the fixed width of the insert in the present test model. Each of these control devices is implemented upstream of the interaction region. The boundary-layer thickness just upstream of the separation for no control, as estimated from the schlieren images, was $\delta=3.4 \pm 0.04 \mathrm{~mm}$. With respect to the separation location, this test location corresponds to approximately $10 \delta$. The choice of this distance was based on earlier reported studies [13,20,21], which have shown that such controls perform most effectively between 6 and $20 \delta$. The boundary-layer thickness was also estimated based on length Reynolds number $R e_{x}$ for turbulent flows $(3.48 \mathrm{~mm})$ with a corrected value of $3.56 \mathrm{~mm}$ for compressible flows, as suggested by Van Driest [37]. However, such an estimate may differ from the boundary-layer thickness calculated from velocity profile by $3 \%$ [38].

The tunnel stagnation pressure $P_{o}$ was acquired using a 200 psia transducer with $\pm 0.1 \%$ of full-scale accuracy, whereas the static pressure measurements such as $p_{w}$ and $p_{\infty}$ were acquired using 30 psia transducer with $\pm 0.04 \%$ of full-scale accuracy. The pressure transducers were calibrated using a five-point calibration procedure before the beginning of the experiments. Further, a single-point check calibration was performed each day to check for any drift in error. The uncertainties in the pressure measurements were estimated using a statistical approach based on repeatability tests. The estimated uncertainty in measurement of total pressure was $\pm 1.4 \mathrm{kPa}$, and that for static pressure measurements was $\pm 0.7 \mathrm{kPa}$. The transducers for

Table 1 Flow characteristics of undisturbed boundary layer

\begin{tabular}{lccc}
\hline \hline Parameter & Value & Parameter & Value \\
\hline Boundary-layer thickness $\delta$ & $3.4 \mathrm{~mm}$ & Interaction length $X_{I L}$ & $33 \mathrm{~mm}$ \\
Displacement thickness $\delta^{*}$ & $1.15 \mathrm{~mm}$ & Separation length $X_{\mathrm{SL}}$ & 45 \\
Momentum thickness $\theta$ & $0.34 \mathrm{~mm}$ & Separation criteria $S_{e}^{*}$ & 1.22 \\
Unit Reynolds number $R e$ & $25.257 \times 10^{6} \mathrm{~m}^{-1}$ & Nondimensional interaction length $L^{*}$ & 2.2 \\
$\operatorname{Re}_{\theta}$ & $0.86 \times 10^{4}$ & Skin friction coefficient $c_{f}$ & $2.1 \times 10^{-3}$ \\
\hline \hline
\end{tabular}




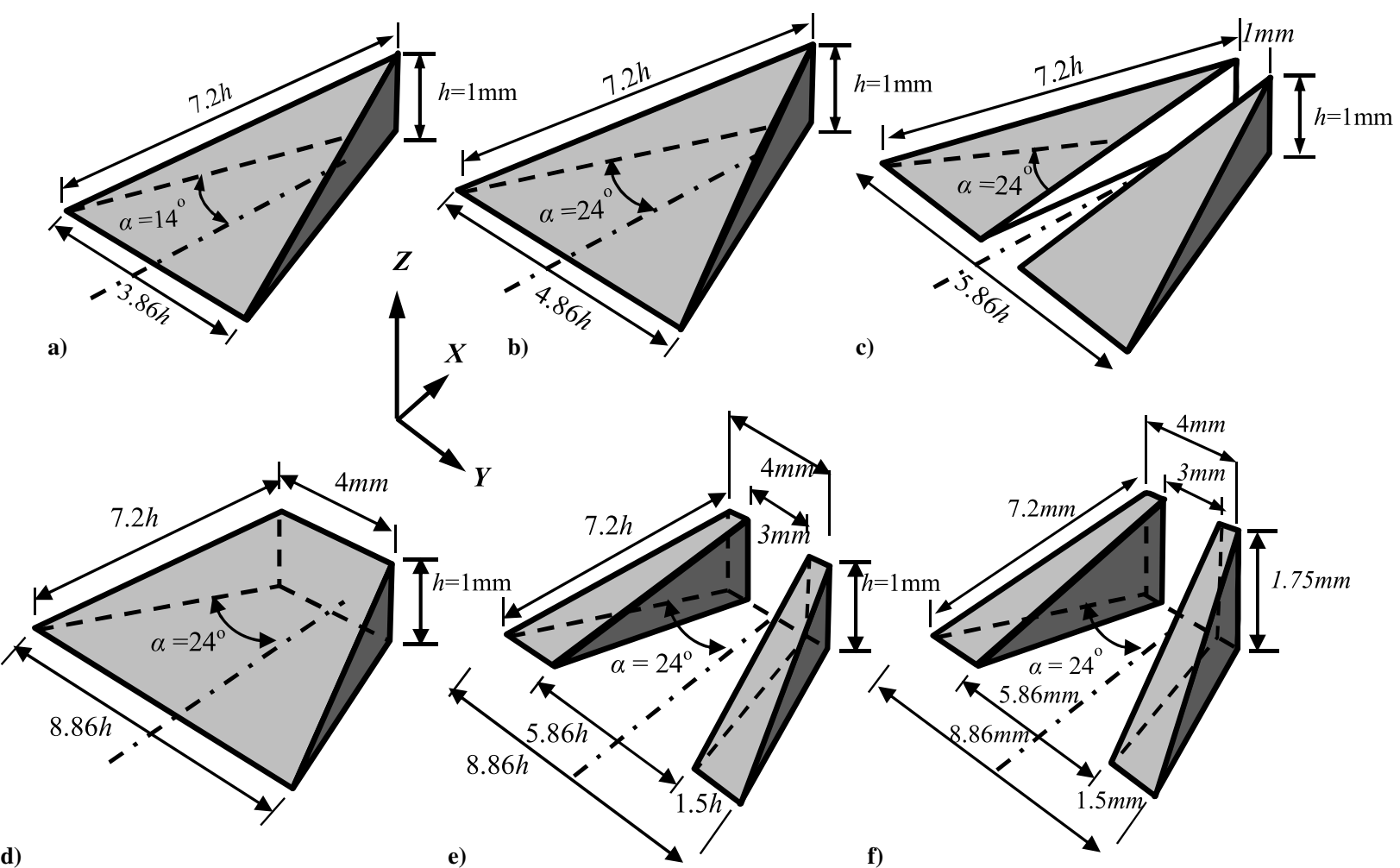

Fig. 3 Schematics of the microramp control configurations: a) Ashill, b) Anderson, c) split Anderson, d) trapezoidal (TRZ), e) ramp-vane (RV1), and f) ramp-vane (RV2: $h / \delta=0.5)$.

unsteady pressure measurements were calibrated statically, and the uncertainty obtained from calibration was found to be within $\pm 1 \%$ of full scale. However, in the intermittent region of separation that is associated with high levels of flow unsteadiness, the average pressure uncertainty is likely to be somewhat greater. The repeatability of the peak rms values in the interaction region was found to be roughly within \pm 0.04 . The rms of the wall pressure fluctuations upstream of the interaction and normalized by the freestream dynamic pressure $q_{\infty}$ for the present tests was $2.18 \times 10^{-3}$. Furthermore, the frequency of the temporal scales of the incoming boundary layer are much higher $\left(U_{\infty} / \delta=150 \mathrm{kHz}\right)$ than the filter cutoff frequency (of $20 \mathrm{kHz}$ ). Because the pressure fluctuations in the shock-wave/ boundary-layer interactions (SWBLIs) are dominated by relatively low frequencies, this is not considered to be a serious limitation. For the incoming undisturbed boundary layer, the probability density distributions of the pressure fluctuations were essentially Gaussian with skewness and kurtosis values equal to 0.03 and 3.02, respectively. The $\sigma_{w} / P_{w}$ value for the incoming boundary layer was $0.0037 \pm 2 \%$.

\section{B. Signal Conditioning and Data Acquisition System}

The wall static pressures in the region of interaction were measured using both electronic pressure ESP scanners and Kulite transducers. The model had 22 (P1 to P22) mean static pressure ports, which were measured using Pressure Systems ESP-16HD 16-port scanners. These scanners were calibrated in situ using a Druck calibrator Model DPI-610. An eight-channel signal conditioner module (SCXI 1520) from National Instruments is used for acquisition of the analog signals from the pressure scanners. The analog signals are then digitized using a 16-channel 16-bit A/D card (NI 6036) that has a maximum sampling rate of $200 \mathrm{kS} / \mathrm{s}$. The present data were acquired at $500 \mathrm{~Hz}$, with 500 samples taken for each port location. This resulted in an averaging time of $1 \mathrm{~s}$. The unsteady wall pressure fluctuations were measured using 12 fast piezoresistive Kulite model XCQ-093 M-screen transducers at locations marked K1 to K12 in Fig. 1c. The presence of the protective screen limits the frequency response of these transducers to $50 \mathrm{kHz}$. The Kulite transducers have a pressure-sensitive area of $0.071 \mathrm{~cm}$ and an outer casing diameter of
$0.26 \mathrm{~cm}$. The transducers were not flush-mounted on the base plate. Instead, a small orifice (of $1 \mathrm{~mm}$ length and $0.5 \mathrm{~mm}$ diameter) connects the transducer to the base flow. This configuration results in an estimated resonance frequency of $68.75 \mathrm{kHz}$. According to the manufacturer's specifications, these transducers have a natural frequency of approximately $250 \mathrm{kHz}$. The sensitivity of the transducers is typically $3-4 \mathrm{mV} / \mathrm{psi}$. These transducers were calibrated statically. The transducer data were acquired using truly simultaneous acquisition card NI4495 DC series (with 24-bit resolution) at a sampling frequency of $50 \mathrm{kHz}$. Each sensor was powered by dc power supply, and the signal was passed through an amplifier and a signal conditioner. A low-pass filter of $20 \mathrm{kHz}$ was applied postacquisition during data processing. For each transducer channel, 200 records of 4096 were acquired, yielding a total of 819,200 data points per channel per tunnel run. For spectral analysis, a 4096-point narrowband fast Fourier transform was performed and later averaged for 200 records, giving a frequency resolution of $12.2 \mathrm{~Hz}$.

Color schlieren technique (using a vertical-banded RGB filter at the knife-edge location upstream of the camera) has been used to capture the flowfield interaction. Palflash 501 with spark duration of $500 \mathrm{~ns}$ and pulse energy of $6 \mathrm{~J}$ was used as light source. The Z-type setup uses 3.0-m-focal-length spherical mirrors to collimate and refocus the illumination source at the knife-edge location. The color filter was adjusted in a way that the green color represents refractive index gradient near zero. Schlieren images were captured using a Nikon 1X digital camera with a $300 \mathrm{~mm}$ lens. The exposure time was set at $125 \mu \mathrm{s}$.

\section{Results and Discussions}

\section{A. Schlieren Flow Visualization}

Figure $4 \mathrm{a}$ and Figs. $4 \mathrm{~b}-4 \mathrm{f}$ show the color schlieren images of a $14 \mathrm{deg}$ incident shock-induced interaction with no control and the modified interactions with control, respectively. For the test case with no control (Fig. 4a), the flowfield upstream of the interaction shows a series of weak waves (inclination of $30 \mathrm{deg}$ or Mach waves) emanating from 1) the edges of the dummy inserts fabricated for the no control or the baseline case and, 2) the junction between the two 

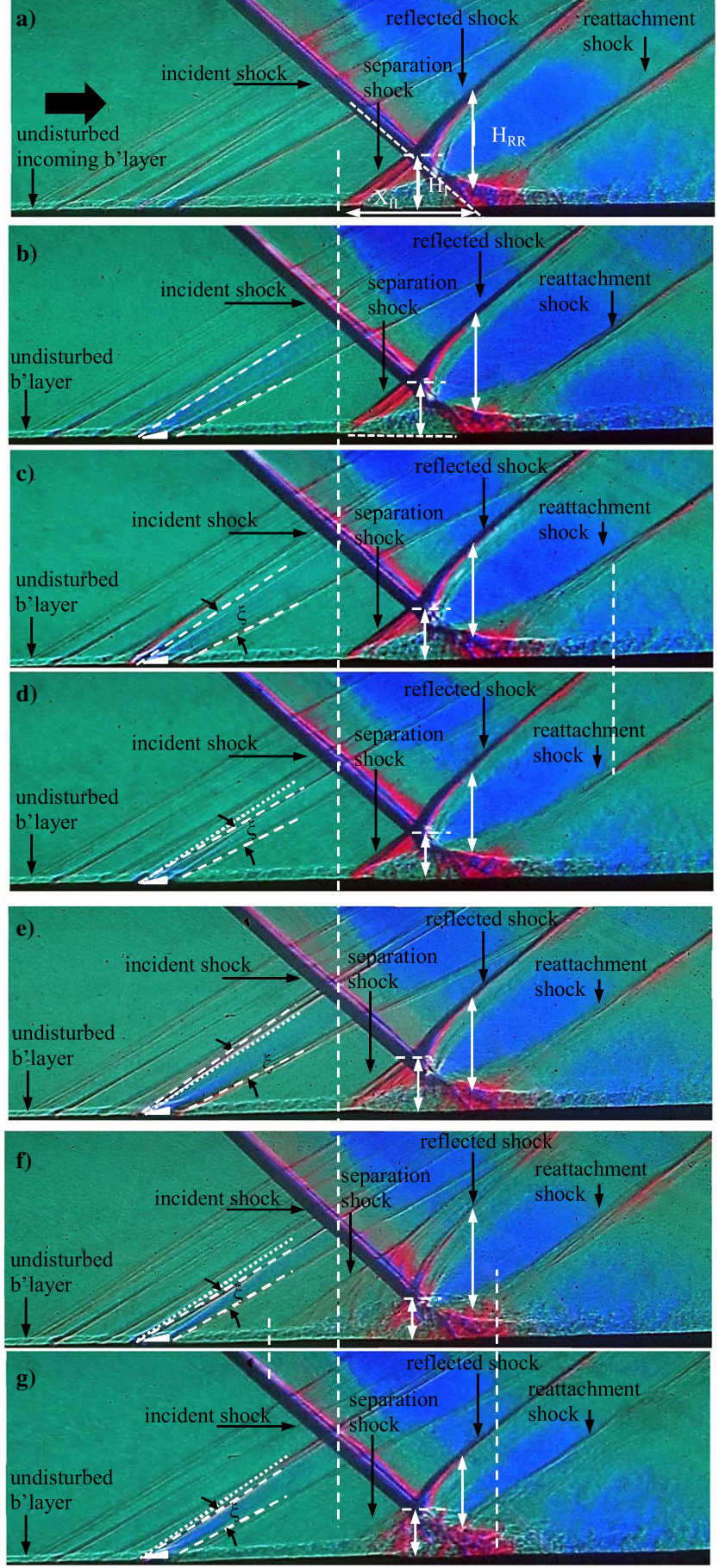

Fig. 4 Color schlieren images of the incident shock-induced separation: a) no control, b) Ashill, c) Anderson, d) split Anderson, e) TRZ, f) RV1, and g) RV2; 14 deg SG; $X=10 \delta$.

insert locations where the leading edge plate meets the base plate, as seen in Fig. 1c, and do not seem to introduce perturbations in the incoming undisturbed boundary-layer. Surface oil visualization tests conducted later further confirm that these weak disturbances do not introduce any local or spanwise interference that may influence the flow development in any way. It may further be noted that these upstream conditions remain the same for all the VG configurations tested. The shock-shock interaction seen is of type 1 [39]. An expansion fan emanating from the shoulder of the $\mathrm{SG}$ is also clearly seen.

The implementation of control devices upstream of the interaction are seen to introduce local perturbations in the form of a compression, followed by an expansion, and then finally a recompression (Figs. 4b-4g). Both the compression and recompression shocks are marked by dashed lines ahead of and behind each control device, respectively, to indicate the extent of local interaction induced by each device configuration. The local compression shock angle ahead of the Anderson device is taken as the reference and is superimposed on the schlieren images of all the other device configurations (Figs. 4d-4g) as a dotted line for comparison. Compared to the Anderson device, the split Anderson shows a relatively much smaller compression shock angle, indicating a reduction in the severity of the localized SWBLI. A similar observation can also be made for RV1 and RV2 devices. The TRZ configuration, on the other hand, with a wider spanwise and base dimension (Fig. 3d), shows a relatively higher compression shock angle (also seen as a larger interaction angle $\xi$ ), indicating a stronger localized interaction ahead of the device. A reduction in the compression shock angle ahead of each device, relative to that of the Anderson device, is an indication of the reduction in severity of the localized SWBLI in the immediate vicinity ahead of the control device. Further details on such local variations in the flow development processes will be discussed in Sec. III.F.

The overall changes introduced to each interaction by various control devices is broadly assessed based on the changes observed in the separation shock-foot location (marked by vertical dashed lines). Because the schlieren images are instantaneous images of the spanwise integrated flow, only a qualitative comparison based on individual snapshots is made. Although the shock locations do show changes from snapshot to snapshot, the changes observed are, however, less than $4 \%$. The measure of the changes introduced for each case is assessed from the changes seen in the interaction point height $H_{I}$, the distance $H_{R R}$ between the reflected and reattachment shocks (as shown by a vertical double headed arrows), and $X_{I L}$ (as defined in Fig. 4a) and are listed in Table 2 for comparison. The uncertainty in measurement of each of the preceding is approximately \pm 5 , \pm 4 , and $\pm 3 \%$, respectively. Relative to no control, for all the initial three control configurations considered, the separation shock-foot location seems to have slightly moved back (Figs. $4 \mathrm{~b}-4 \mathrm{~d}$ ), as will also be seen later in the mean pressure distributions. The split-Anderson device shows a maximum reduction in the heights $H_{I}$ and $H_{R R}$. The separation shock-foot location for TRZ, however, seems to remain almost unchanged, with minimal changes in $H_{I}$ and $H_{R R}$. The RV1 and RV2 devices, on the other hand, introduce major modifications to the overall interaction. Although for the RV1 device, the separation shock foot is seen to move significantly upstream (Fig. 4f), the RV2 control, on the other hand, shows a significant downstream movement of the separation shock-foot location (Fig. 4g), relative to no control. It may, however, be pointed out (and as will be seen from the surface oil pictures later) that the flow in the region of separation is highly three-dimensional, especially in the case of RV1 and RV2 control configurations, and hence interpreting changes in shock-foot location from schlieren images is only qualitative and should be used in conjunction with mean wall pressure distributions discussed later in Sec. III.C. $H_{I}$ and $H_{R R}$ are also seen to get significantly reduced, indicating effective control modifications.

Table 2 Variation of parameters $H_{I}$ and $H_{R R}$ as a percentage of $X_{I L}$

\begin{tabular}{lccccccc}
\hline \hline Parameter & No control & Ashill & Anderson & Split-Anderson & Trapezoidal & RV1 & RV2 \\
\hline$H_{I}$ & 0.52 & 0.52 & 0.52 & 0.37 & 0.52 & 0.37 & 0.37 \\
$H_{R R}$ & 0.89 & 0.89 & 0.89 & 0.74 & 0.89 & 0.89 & 0.67 \\
$X_{I L}$ & 1.0 & 0.96 & 0.96 & 0.92 & 1.0 & 1.63 & 0.71 \\
\hline \hline
\end{tabular}




\section{B. Surface Oil-Flow Visualization}

After discussing the off-surface flowfield modifications, let us study the changes introduced in the surface flow topologies with control. It may be noted that, for each surface oil-flow picture shown, a front view is given, and a corresponding schematic is presented, which is developed based on the flow features observed (Fig. 5). It can be seen that the flow topology for the entire interaction as well as the region downstream of the interaction is very clearly captured. The surface oil visualization tests with the dummy insert and modular plates ahead of the VG insert with and without control show no disturbance upstream or downstream of these modular plates in the flow topology, which indicates that there is no interference introduced whatsoever (spanwise or streamwise) from these inserts that maybe be influencing the flow development in any way. Each picture in Fig. 5 also shows the Kulite transducer locations (dashed line), which are off-center from the circular dotted line along the centerline (mean pressure port locations) by $5 \mathrm{~mm}$. The flow topology shown in the schematics to the extreme right is developed based on the spanwise variation in the flow pattern observed in the region of interaction and about the model centerline. Beyond a certain spanwise distance, the separation line begins to slightly curve downstream. The latter alters the way that the flow structures from the devices interact with the reverse flow in the separation region, as a result of which the flow pattern is no longer able to reproduce the flow separation topology observed in the flow pattern about the centerline. The dark color used in the corresponding schematics depicts the separation region. In the surface oil pictures, the black and the white dashed lines mark the separation and reattachment lines, respectively, and are captioned only in Fig. 5a. The vertical white dotted lines show the lines of oil accumulation beyond the reattachment location and indicate the striation pattern on the flat plate.

Relative to no control (Fig $\underline{5 a}$ ), all control devices are seen to introduce spanwise variations in the form of corrugations in the separation line. This is caused due to the counter-rotating streamwise vortices, generated in the wake of these devices, interacting with the reverse flow in the separation bubble [40]. However, the size and strength of these vortices are dictated by the configuration of each device (Lee et al. [27]), which in turn controls the shape and amplitude of these corrugations. For example, splitting the conventional Anderson-type configuration (Fig. 5b), a splitAnderson configuration (Fig. 5c) breaks the relatively smaller amplitude of the corrugations, observed for Anderson type, into sharper spikes with almost no noticeable change in the shape of the reattachment line. An earlier study [27] has shown that, although this configuration generates much stronger vortices compared to the Anderson configuration, the split results in a larger velocity deficit region downstream, which offsets its benefits at larger control distances. This means that the control location for improved effectiveness of a control device may vary for different control configurations. The TRZ device also shows almost similar flow separation topology as that seen for the Anderson-type device, except that the amplitude of the corrugations seems to have become relatively larger (Fig. 5d). In contrast, the RV1 device moves the separation location upstream, as was also seen in schlieren images, showing a single large corrugation (Fig. 5e). Several repetitions of oil visualization tests were conducted to verify the repeatability of this flow pattern. This included realigning of the RV1 insert, removing it and conducting tests with another VG configuration insert, and finally putting the RV1 insert back in position. Although none of the other VG configurations ever showed a skewed separation line as seen for RV1, the RV1 always showed this flow pattern. A somewhat similar flow separation topology was also observed by Lee at al. [27] from their large-eddy simulation (LES) and Reynolds-averaged Navier-Stokes study for the ramped-vane device of $h=0.34 \delta$ when placed in an array. In fact, the length of the corrugation (formed between adjacent devices) was observed to increase as the device was moved farther away from the shock location. In the present case, the three-dimensional nature of the base interaction seems to have perhaps further aggravated this effect, resulting in a highly skewed effect on the downstream flow in the presence of neighboring VGs. Lee et al. [27] further noticed that increasing the intervane spacing or
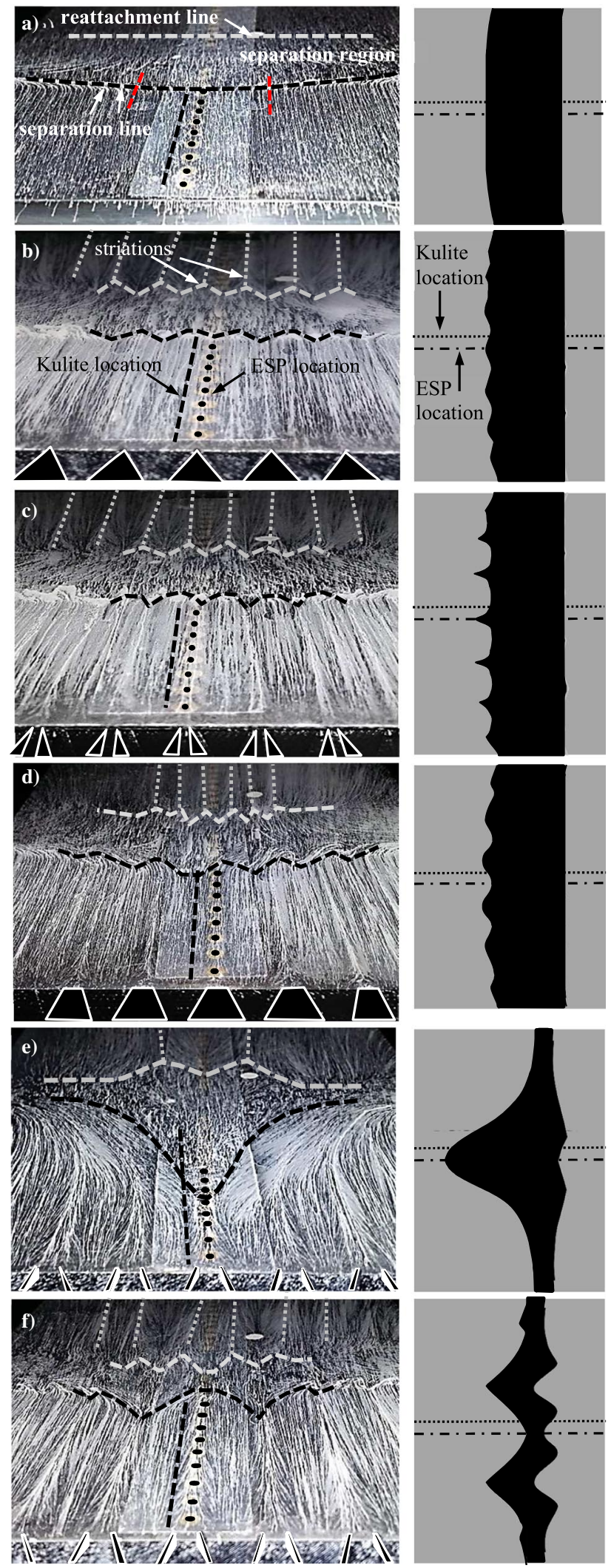

Fig. 5 Surface oil visualization pictures: a) no control, b) Anderson, c) split Anderson, d) TRZ, e) RV1, and f) RV2; $14 \mathrm{deg}$ SG; $X=10 \delta$.

the distance between the CRVs seems to be beneficial in reducing the upwash induced by the vortices on each other, which is much higher in the case for the conventional delta ramp and the split-Anderson type devices. However, doing so increased the separation considerably along the device centerline. Increasing the height of 
RV1 to form RV2 is seen to result in the formation of majorly two corrugations. Downstream of the device and along the centerline, the separation location is considerably pushed downstream, resulting in a significantly large reduction in the extent of separation. In the detailed study conducted by Lee et al. [27], the spanwise view of streamwise vorticity contours showed that the size of the vortices shed in the wake of a higher ramp-vane height device are much larger. As a result, the intervane spacing seems to be critical where the closer proximity of a neighboring device can cause an early upwash of the CRVs shed from them.

Another important flow feature not reported before (other than that reported by Verma and Manisankar [41]) is the well-defined striation pattern downstream of reattachment location, which gives an idea about the impression the CRVs can leave on the entire interaction. Such a striation pattern is observed only for the control cases, indicating that the origin of these in the present scenario is not due to Goertler vortices associated with reattaching boundary layers. It is interesting to see that each striation downstream of flow reattachment is formed exactly in line along the center of each interdevice spacing. Such a striation pattern downstream of reattachment was not observed by Souverein and Debiève [12], although the array of air jet vortex generators in their case also generate pairs of counter-rotating vortices upstream of the separation bubble. These tests were conducted at Mach 2.3 on an incident shock-induced separation using a $9.5 \mathrm{deg}$ SG with an incoming boundary-layer thickness of $10 \mathrm{~mm}$. Further, the reattachment line in their experiments did not show any corrugations, unlike the present study. They reason their observation to the lifting off of the longitudinal vortices over the interaction by the separation bubble and later getting destroyed by the unsteady processes occurring in the interaction region. The present observations indicate that the longitudinal vortices do lift off during separation, but they do not get destroyed thereafter. In fact, the placement of the striations with respect to the VG locations and the direction of the streamlines about each striation and those immediately downstream of reattachment suggest that, on reattachment, these longitudinal vortices impinge on the plate, forming CRVs of opposite sign, which results in the observed striation pattern. The interaction of the flow reversing at reattachment location and the impinging CRVs then results in the formation of a corrugated reattachment line.

\section{Streamwise Mean and Fluctuating Pressure Distributions}

Figures 6 and 7 show the streamwise distribution of mean pressure $P_{w} / P_{a}$ from ESP $\overline{\text { scanners }}$ (placed along the centerline, Figs. $\underline{6 a}, \underline{6 b}$, $\underline{7 \mathrm{a}}$, and $\underline{7 \mathrm{~b}}$ ) and Kulite transducers (placed $5 \mathrm{~mm}$ off-center, Figs. $\underline{6 \mathrm{c}}$ and $7 \mathrm{c}$ ), whereas Figs. $6 \mathrm{~d}$ and $7 \mathrm{~d}$ show the associated standard deviation distributions $\sigma_{w} / P_{a}$ for the latter in the region of interaction with and without control. For clarity, Figs. $6 \mathrm{~b}$ and $7 \mathrm{~b}$ show the zoom of separation region from Figs. $6 \mathrm{a}$ and $7 \mathrm{a}$, respectively. The mean pressure distributions along the centerline in Figs. $\underline{6 a}$ and $\underline{6 b}$ clearly show that, relative to no control, the separation location is pushed back by one transducer location for all three control configurations. It is also interesting to see that the initial rise in wall pressure at separation (as shown by drawing a dashed tangent) seems to get reduced slightly as we move from Ashill to Anderson and, finally, to split-Anderson configuration. The mean pressure distribution based on Kulite data (Fig. 6c) shows a slightly different distribution compared to Fig. $6 \mathrm{~b}$, although the overall trend remains almost similar, indicating that the flowfield induced by the control devices at separation is three-dimensional, as was also seen in Fig. $\underline{5}$. It maybe
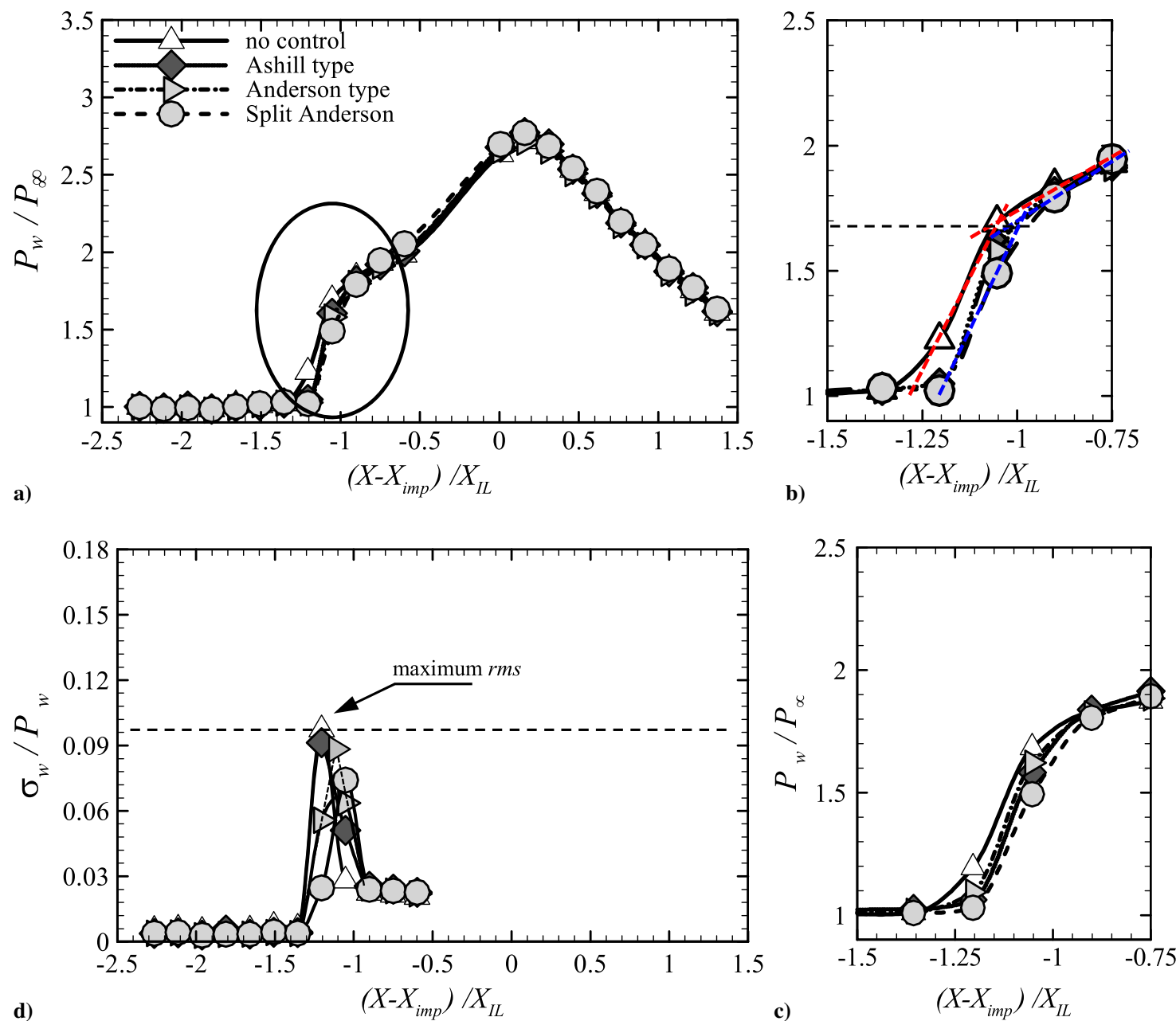

Fig. 6 Comparison of streamwise distribution of mean pressure and its corresponding standard deviation with and without control for a-d) Ashill and Anderson-type control devices: VG location $\left(X-X_{\text {imp }}\right) / X_{I L}=-2.4$. 

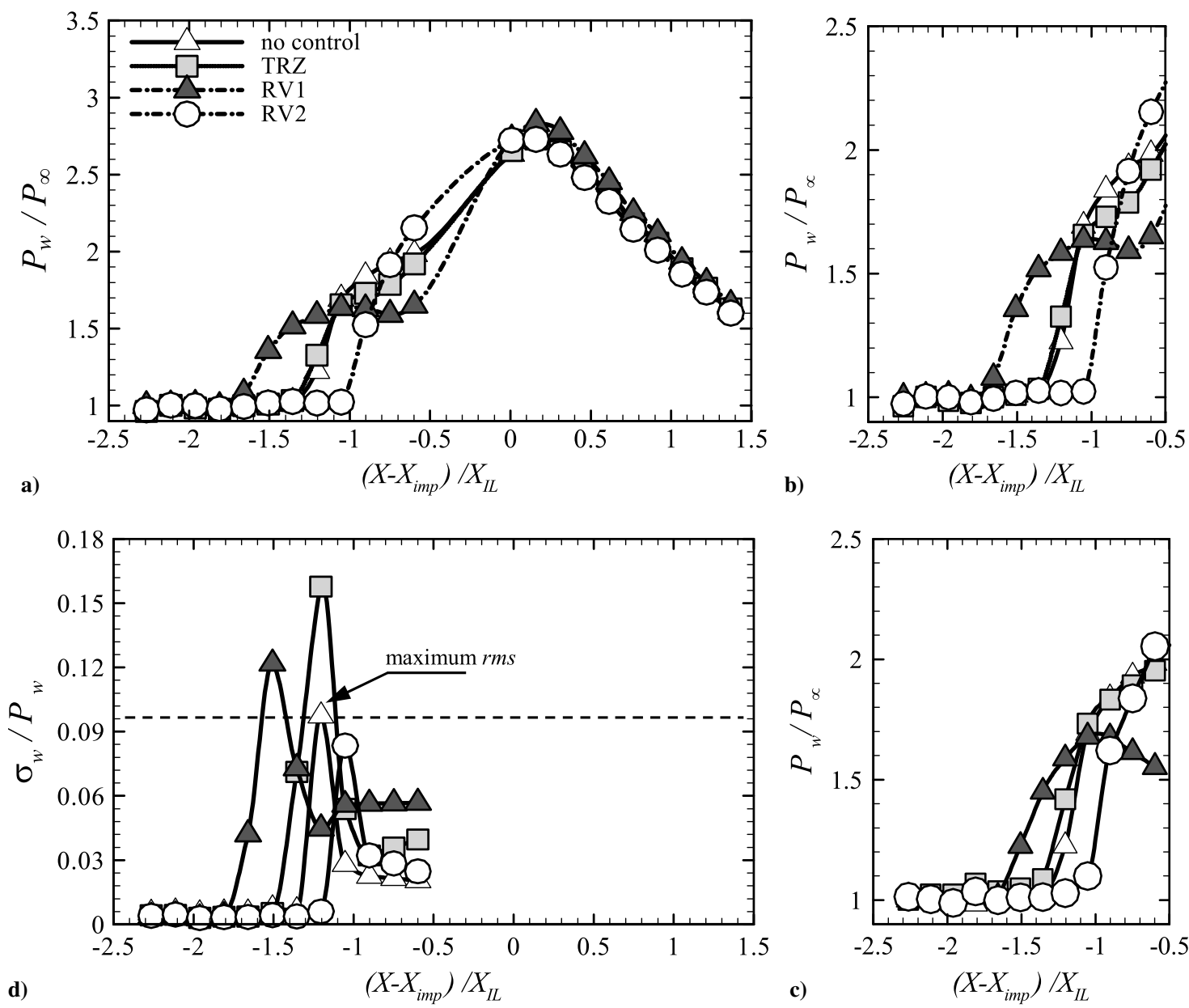

Fig. 7 Comparison of streamwise distribution of mean pressure and its corresponding standard deviation with and without control for a-d) trapezoidal and ramp-vane type devices: VG location $\left(X-X_{\text {imp }}\right) / X_{I L}=-2.4$.

indicated here that, because the Kulite transducers are placed $5 \mathrm{~mm}$ off-center of the control device centerline, this measurement location corresponds to a device off-center location of almost $100 \%$ span. Referring to the surface oil pictures for these three control configurations, this measurement location corresponds to the trough of the separation line corrugation (Figs. $5 \mathrm{~b}$ and $\underline{5 \mathrm{c}}$ ).

Most of the earlier studies $[3,20,42]$ on $\overline{M V G}$ effect on the interaction unsteadiness have focused on the unsteadiness of the separation shock or, in particular, the separation shock foot. A good measure of the reduction in the separation shock-foot unsteadiness has been related with the reduction in maximum rms value of wall pressure fluctuations in the region of separation [3] with and without control. A similar approach is followed for the present tests, and the data for streamwise distribution of the rms values (associated to data in Fig. 6c) are plotted in Fig. 6d. The standard deviation distribution in the intermittent region shows a reduction in maximum rms value, relative to no control, in going from Ashill device (1\%) to the Anderson configuration (5\%) and, finally, to the split-Anderson device, which shows $24 \%$ reduction. Beyond the intermittent region of separation, the fluctuation levels for all test cases are similar. It is interesting to note that even though the measurement location for rms data is located off-center of the device centerline at $100 \%$ span, the control devices are still able to reduce the shock unsteadiness significantly. It has been reported earlier [20] that the effects of control devices on the maximum rms value are not localized on the separation shock foot but are also affected in the spanwise direction in addition to that along the device centerline. Based on these observations, Giepman et al. [20] hypothesized that, by breaking up the separation bubble into cellular structures, as seen by the corrugations in the separation and reattachment lines as well as the striation pattern downstream of reattachment (Fig. $\underline{5}$ ), the degrees of freedom of the reflected shock wave are reduced, which consequently reduces the shock unsteadiness.

Other control devices such as the ramp-vane configurations are seen to introduce major changes to the overall interaction (Figs. 7a-7d). For the trapezoidal configuration, along the centerline, the mean pressure distribution shows the separation location to remain similar to that for no-control case (Figs. 7a and 7b) but shows a reduction in the pressure after separation. The RV1 device, on the other hand, shows significant upstream separation relocation (by $40 \%$ ), whereas RV2 shows a downstream movement (by 21\%) of separation location with minimal changes, if any, in pressure distribution beyond the $X_{\text {imp }}$ location (Fig. 7a). Because of the highly three-dimensional flow induced by these devices, especially for RV1 and RV2, as seen in Figs. 5d-5f, the mean pressure distribution from Kulite transducers shows considerable changes relative to that along the centerline (Fig. 7c). It may be noted here that, for these three devices, the Kulite locations correspond to almost $50 \%$ of device span (due to the higher spanwise width of these devices, Fig. $\underline{3}$, compared to the previous set of devices). For this spanwise measurement location, the TRZ shows a slight upstream movement of separation location, whereas the pressure rise after separation for it remains similar to the no-control case, unlike that seen in ESP pressure distribution (Fig. 7b). For RV1, the separation location is seen to be slightly downstream of what is seen in Fig. 7b, whereas for the RV2 case, the separation location is seen to have moved slightly upstream of that seen in Fig. 7b. As seen in surface oil pictures, the flow is highly three-dimensional, especially for RV1 and RV2, and hence shows large variations in pressure distribution along the span. Looking at the associated streamwise rms distributions, relative to no control, the TRZ configuration shows a significant rise in the maximum rms value (by $62 \%$ ), followed by RV1 ( $25 \%$ increase), and 


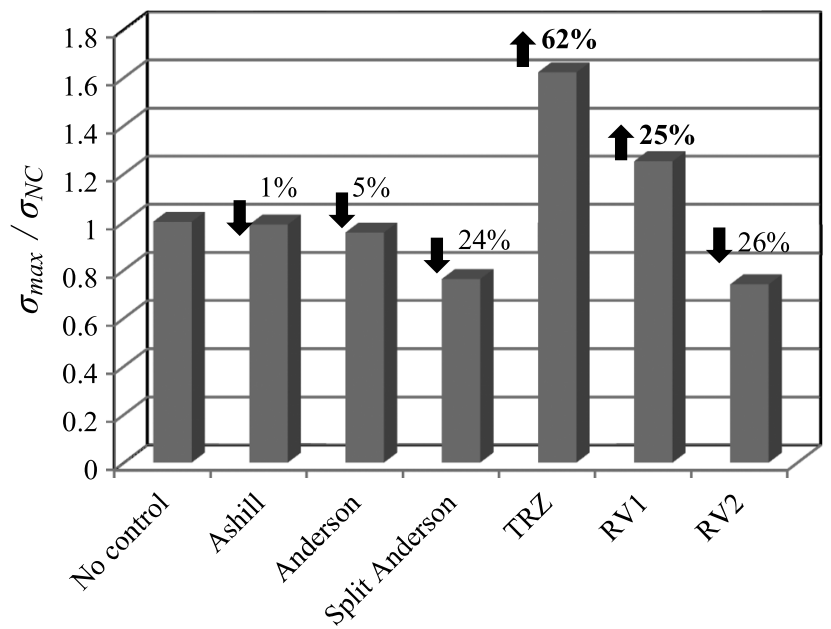

Fig. 8 Comparative bar chart showing the variation in maximum rms values in the region of separation for all control devices tested. The bars with upward arrows show increase in rms value.

finally RV2, which shows maximum reduction of $26 \%$ (Fig. 7d). Figure 8 shows a comparative bar chart of the maximum rms values $\sigma_{\max } / P_{w}$ in the region of separation/reflected shock foot for all the control devices tested. Here, the maximum rms value $\sigma_{\max }$ for each device is nondimensionalized by the maximum rms value for no control $\left(\sigma_{\mathrm{NC}}\right)$. It is clearly seen that the split-Anderson and the RV2 devices show significant reduction in maximum pressure fluctuations of the separation shock foot.

\section{Power Spectra}

Spectral analysis of the dynamic pressure signal was also conducted to look into the temporal characteristics of the wall pressure fluctuations in the region of separation. The normalized power spectral density function, $G(f) \cdot f / \sigma_{w}^{2}$, is used because it helps to eliminate the relative differences in the magnitude of the fluctuating pressure signals and enhances the frequency contributions $[3,9,23]$. However, to bring out the effect of control on the amplitude of fluctuations, relative to no control, the quantity $G(f) \cdot f$ is nondimensionalized with maximum rms value for no control $\sigma_{\mathrm{NC}}^{2}$ (Fig. 9a). The quantity $G(f) \cdot f$ is also nondimensionalized with the respective maximum rms value $\sigma_{w}^{2}$ for each individual case for comparison as done traditionally and is shown in Fig. $9 \mathrm{~b}$.

For no control, the spectra at the location of maximum rms in the region of separation show a dominant frequency centered approximately around $0.35 \mathrm{kHz}$ (Fig. 9a). In terms of dimensionless shock frequency $S t=f_{s} l / U_{\infty}$, this value correspond to 0.027 , where $f_{s}$ is the characteristic shock frequency, $l$ is the length of separation, and $U_{\infty}$ is the external or freestream velocity. The Strouhal number of 0.027 observed for the baseline case here is within the range of 0.025 0.04 reported for incident shock interactions [43]. With control, significant variation in the amplitude of pressure fluctuations at the location of maximum rms is observed. Both Ashill and Anderson devices are seen to bring down the amplitudes only slightly. On the other hand, the split-Anderson and RV2 devices show significant reductions, whereas TRZ and RV1 show a considerable increase. These variations in the amplitude of pressure fluctuations are in conformity with the variation in maximum rms value seen in Fig. 8. It may be noted that, for the split-Anderson and RV2 configurations, in addition to the reduction in amplitude of fluctuating pressure loads, a shift in dominant frequency to relatively higher values (of approximately 0.4 and $0.6 \mathrm{kHz}$, respectively) is also observed. Earlier studies [3] have shown that a reduction in maximum rms value that is accompanied by a shift in the dominant frequency of pressure fluctuations to a higher value is primarily attributed to a weaker separation shock due to the vortex generators and an increased jitter in the separation shock motion (shorter periods). It is interesting to note that, when the quantity $G(f) \cdot f$ is nondimensionalized with the respective maximum rms value $\sigma_{w}^{2}$ for each individual case, the variation in amplitude of fluctuations almost disappears (except for RV2), and the spectra shown in Fig. 9b exhibit a similarity despite differences in control device configurations and the three-dimensional effects introduced by each of them in the interaction region.

\section{E. Process of Flow Development from Various Vortex Generator Configurations}

The present subsection discusses how the flow development process from various VG configurations varies based on the VG design. The discussion is partly based on the observations made from the present surface flow visualization results and partly from the experimental and computational results reported earlier in literature. Additional study, however, needs to be conducted to validate some of the conclusions made in the present section, which are speculative at the moment. Figure 10 shows zoomed pictures of the surface oil tests and the associated flow sketches developed for individual test cases for the sake of discussion. The surface oil pictures show that, in the vicinity of the control device, the surface streamlines in the device wake experience a flow turning toward the control centerline immediately after negotiating the control device. This flow pattern is typical of the formation of counter-rotating vortices (CRVs) developing in the streamwise direction that are formed as a result of flow separating from the slant sides [44] and rolling up into vortices along each side of the device. However, it is worthwhile to mention that the location where these CRVs, formed from each side of the device, meet along the control centerline is seen to vary based on the
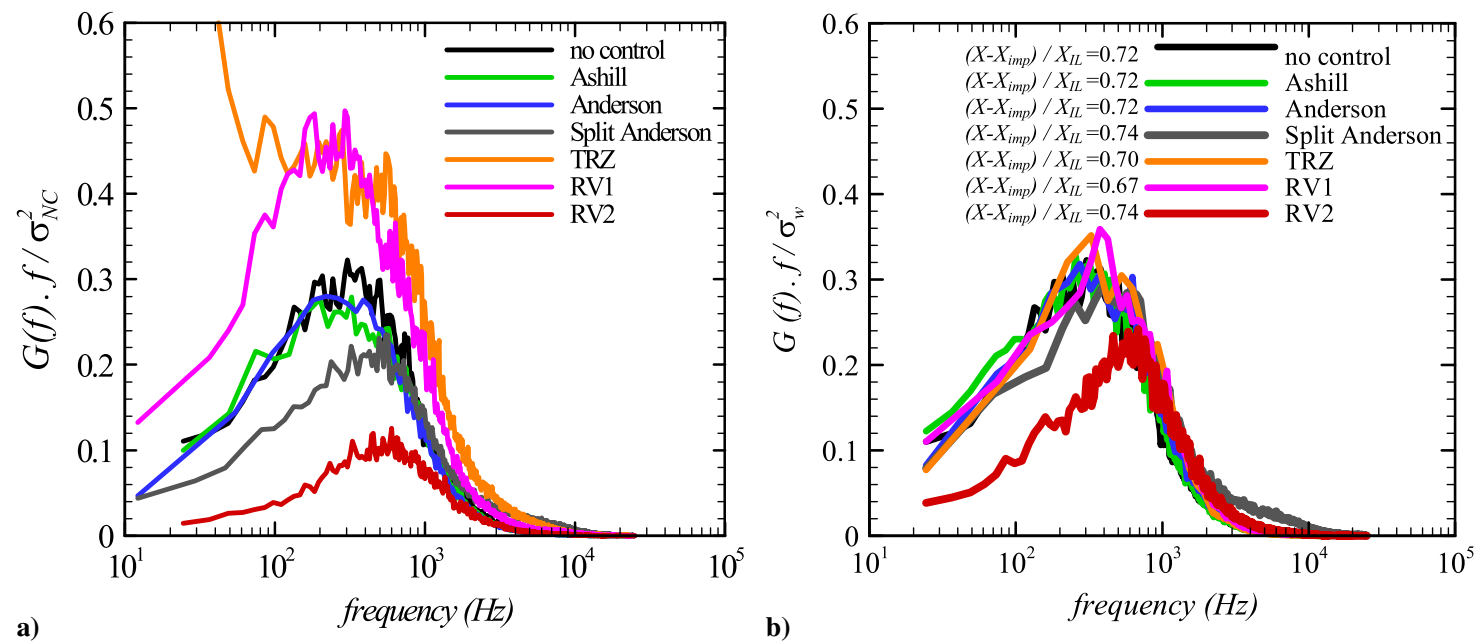

Fig. 9 Comparison of spectra at the location of maximum rms a) $G(f) \cdot(f)$ non-dimensionalized by no control maximum rms value $\sigma_{\mathrm{nc}}$ and, b) $G(f) \cdot(f)$ non-dimensionalized by the $\sigma \max$ value for each device. 

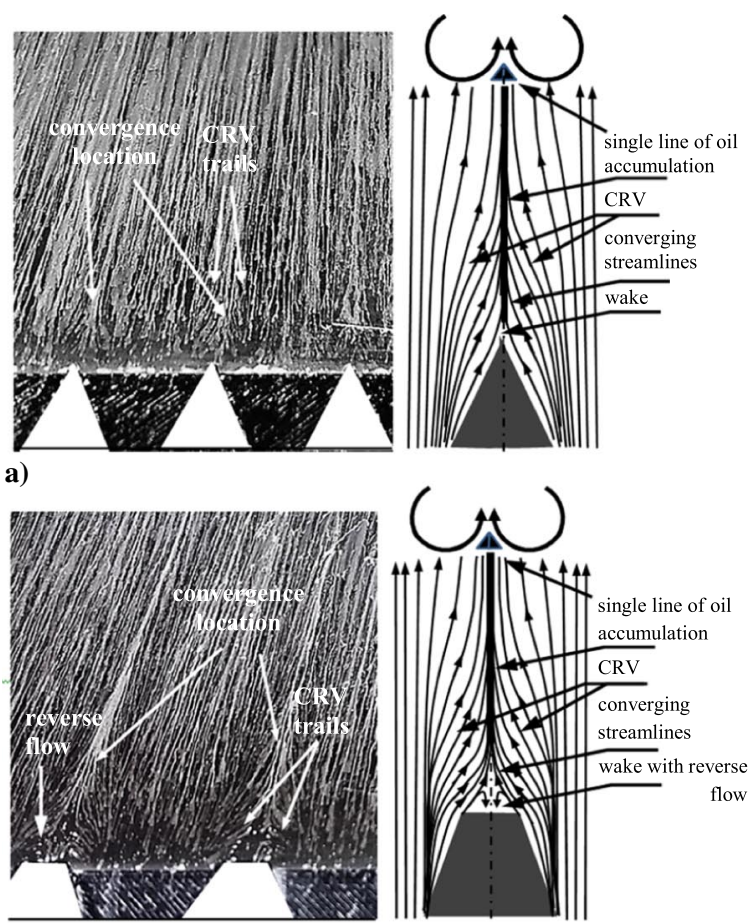

b)
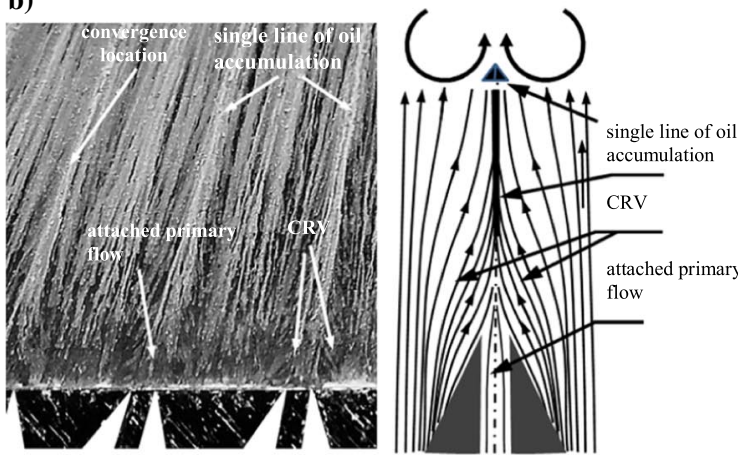
accumulation

c)
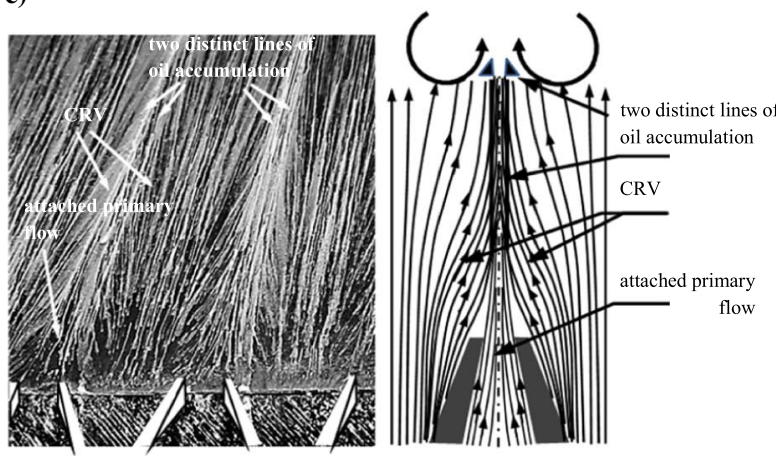

d)
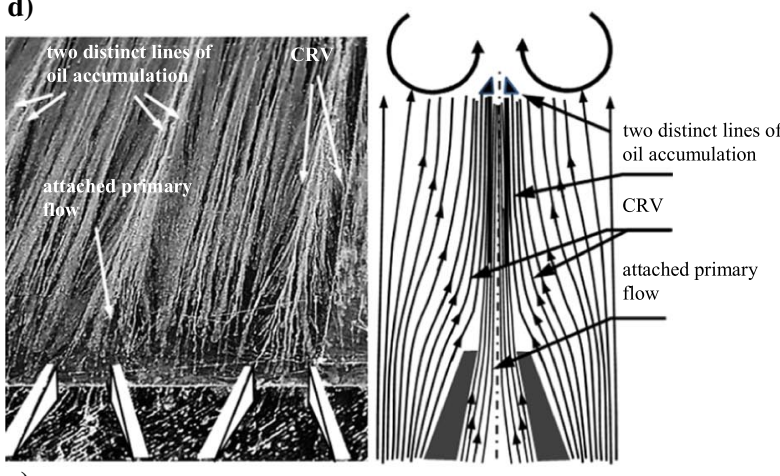

e)

Fig. 10 Close-up of the surface oil visualization pictures in the wake of the control devices and the schematic of the flow development: a) Anderson, b) TRZ, c) split Anderson, d) RV1, and e) RV2. control configuration design. In the discussion of these flow sketches, it may also be noted that the beginning of the solid black line in the wake and along the centerline of the control devices roughly indicates (and not to scale) the location of the merger of the CRVs. A variation in the location of its formation (i.e., CRV merger location) is indicative of the variation in flow development process in the immediate vicinity of each device configuration. The discussion attempts to correlate the importance of CRV merger location, if any, on the effectiveness of the device in controlling the extent of separation.

Let us first discuss the flow topology from control devices such as the Anderson and trapezoidal configurations (Figs. 10a and 10b). Previous computational [45] and experimental pressure-sensitive paint [46] studies have shown that, because of the localized SWBLI induced by the control devices such as the Anderson configuration, a high-pressure region exists ahead of the leading edge and on the forward face of the device ramp. Thereafter, the pressure on the forward face of the ramp starts to decrease as the flow moves toward the ramp trailing edge due to the flow spilling from the slant edges of the device [46]. This means that the flow moving up the ramp surface and expanding into the device wake at the vertex and that spilling from the edges of the device play a dominant role in the overall flow structure development. It is also understandable then that any gap or a split provided along the centerline of the control device, as in the split-Anderson or RV configurations, would help to reduce the severity of the shock-induced interaction at the leading edge of these control devices and allow the high-speed flow to pass through, thereby relieving the high-pressure buildup in this local region. A reduction in the compression shock angle formed ahead of each device relative to Anderson and TRZ configurations shows this effect, as indicated in the schlieren images of Figs. $4 \mathrm{~d}-4 \mathrm{~g}$. Such a local pressure-relieving effect experienced immediately ahead of the device leading edge and provided by the introduction of a split in the control device would then also have some implications on the overall flow structure development from these devices.

In the case of the Anderson device, although a small wake is seen to be formed immediately behind the device, a much larger wake both in spanwise and streamwise extent results for the trapezoidal configuration, primarily due to its larger base dimension (Figs. 10a and 10b). The reverse flow in the wake of the latter is also clearly seen in the oil picture, after which the convergence of the streamlines from the CRVs is seen to close the wake (Fig. 10b) along the device centerline. The closing of the wake is then followed by the formation of a single line of oil pigment accumulation slightly downstream (Fig. 10b). This is caused due to the physical nature of the CRVs that feature the convection of streamwise momentum normal to the wall. As a result, alternate regions of upwash (convection away from the surface) and downwash (convection toward the surface) are formed in the streamwise direction that are associated with low and high shear forces, respectively, thereby resulting in the accumulation of oil pigment along the line of upwash. An early formation of an oil pigment line therefore implies a much smaller base wake and an early convergence of CRVs along the centerline. This is clearly indicated in the case of TRZ, for which the formation of the oil pigment line is slightly delayed farther downstream due to a much larger wake as compared to that observed for the Anderson configuration.

The process of flow development, however, changes when a split is introduced along the centerline of the control device (Figs. 10c-10e). It was shown in the LES study reported earlier by Lee et al. [27] that the presence of the split in the Anderson-type configuration helps to allow the primary flow to pass through it and interact with the flow developing from the each half of the device to result in CRVs of relatively higher strengths and larger scale. This additional flow through the split also delays/prevents the CRVs formed downstream of such devices to converge and meet earlier along the centerline. This effect is clearly seen in the surface oil picture of the split-Anderson device and that depicted in Fig. 10c, which shows the primary attached flow passing through the split delaying the convergence location of the CRVs farther downstream compared to Anderson and TRZ configurations (Figs. 10a and 10b). As a result, once again, a single line of oil pigment accumulation is observed for the split- 
Anderson configuration. Such an effect has been shown (Lee et al. [27]) to produce relatively lesser early upwash for this configuration, which allows a much slower liftoff of the CRVs from the surface compared to those caused by the Anderson configurations and hence helps to improve their control effectiveness over larger distances. On the other hand, the ramp-vane configuration has been reported to produce CRVs of much higher strengths (Lee et al. [27]). Compared to the split-Anderson device, this device is reported to delay the liftoff location farther downstream because it has a much wider split (3 mm in our case as compared to $1 \mathrm{~mm}$ for split Anderson) that allows a much higher mass of primary flow to pass through. This effect is also clearly seen in Figs. 10d and 10e, which show two distinct oil pigment lines formed in the wake of each device that are not seen to converge far downstream and up to the point of interaction. An increased distance between the CRVs has been shown by Lee et al. [27] to be beneficial in further reducing the upwash induced by the vortices on each other as compared to that observed for the splitAnderson device. The split has also been reported to produce much wider spanwise variations compared to a baseline Anderson device [25]. Therefore, the size of the split can play a crucial role in preventing an early liftoff, and a careful selection of its dimension can be used to optimize a control configuration, especially from the perspective of selecting an effective control location for a particular application [13,27].

The effect of the split size relative to the device height is also seen for RV1 and RV2 devices for which the split size is same $(3 \mathrm{~mm})$ but the device heights are different. Because the height of the device also influences the size and strength of the CRVs that it generates [27], the size of the split relative to the device height can play an important role in flow structure development for ramp-vane configurations. For example, for RV1, because the device height is small $(h / \delta=0.3)$, the size and strength of the vortices generated from this device would be relatively less compared to that generated from $\mathrm{RV} 2((h / \delta=0.5)$. As a consequence, with a wider split spacing $(3 h)$, the CRVs developing downstream of RV1 would not be able to converge along its centerline even over much longer distances (Fig. 9). This means that the primary flow region would extend over larger downstream distances due to the inability of the CRVs (due to larger spacing between the vanes) to replace it with high-momentum air. Though such a scenario would be beneficial in reducing the upwash induced by the vortices of the CRV on each other, the relatively reduced strength and scale of vortices shed by RV1 over that shed by RV2 [27] perhaps reduces the ability of RV1 device to exercise control effectiveness over larger distances. It has been shown by Lee et al. [27] that placing the ramped-vane devices $(h=0.34 \delta)$ in an array of two or three results in larger regions of velocity deficits, which were seen to relatively increase over longer distances compared to the single ramped vanes. As a result, placing such an array farther away from the shock location resulted in an increase in the separation length (in the form of a large corrugation) along the centerline between the neighboring devices, whereas increasing the intervane spacing (from $s=1 h$ to $4.57 h)$ for a single ramped vane $(h=0.34 \delta$ ) increased the separation considerably along the device centerline. Although speculative at this moment, the combined effect of the larger regions of velocity deficits for RV1 placed in an array and the sensitivity to the intervane spacing perhaps might be the reason why the separation location is observed to move significantly upstream for RV1 in the present study. From this perspective, a closer control location for RV 1 with smaller split spacing (of $2 h$ or $1 h$ ) might prove more effective than the existing one. Further insights into the potentially related flow phenomena responsible for the observed results are only possible through flow diagnostic tools such as particle image velocimetry, which is beyond the scope of the present study. Increasing the size of the device (RV2) not only increases the size and strength of the vortices in its wake but also reduces the speed with which the high-momentum fluid brought in by the CRVs moves the low-momentum air away from the wall $[10,27]$. The latter is helpful in reducing the upwash and improves the device effectiveness over large distances. From this perspective, increasing the device height with nominal spacing between its vanes (split of $1.7 \mathrm{~h}$ as in RV2 case) seems to be beneficial. A thorough study, however, needs to be conducted in the future to carefully access the effectiveness of the split size relative to the device height and the various parameters that define the device configurations from the perspective of achieving significant reduction in extent of separation or separation shock-foot unsteadiness or both.

\section{Conclusions}

An experimental investigation has been conducted to assess the effectiveness of five micro-VG configurations in controlling the extent of an incident shock-induced separation associated with a $14 \mathrm{deg}$ SG in a Mach 2.05 flow. The VG configurations studied include the Ashill, Anderson, split-Anderson, trapezoidal, and rampvane designs. For each of these control configurations, the device height spanned $30 \%$ of the local boundary-layer thickness $(0.3 \delta)$ estimated just upstream of the separation for the no-control case. The control, in the form of an array of VG devices, was implemented $10 \delta$ upstream of the separation location for no control. Additionally, one case of ramp-vane device with height of $0.5 \delta$ (RV2) was also tested. The overall primary objective of the test campaign was to compare and assess the effectiveness of various configurations in controlling 1) the extent of separation, and 2) the associated separation shockfoot unsteadiness. The present study is performed at Mach 2.05 and for only one Reynolds number, and therefore the results need not be generalized for high-speed flows.

All control devices, irrespective of their design configuration, are observed to introduce spanwise variations in the separation line in the form of corrugations. These corrugations originate as a result of the counter-rotating streamwise vortices, generated in the wake of these devices, interacting with the reverse flow in the separation bubble. However, the nature of the vortices generated is dictated by the configuration of each control device, which in turn controls the shape and amplitude of these corrugations. Out of all the five design configurations tested, the RV2 device gives the most encouraging result. A significant downstream relocation of the separation location (by $21 \%$ ) is observed along the centerline of the device that is accompanied by a considerable reduction in the shock interaction point height. For Ashill, Anderson, and split-Anderson devices, relative to no control, the separation location is pushed back by one transducer location along the device centerline. For TRZ, the separation location remains the same as for no control, whereas for RV1, the separation location moves upstream by almost $40 \%$.

With regard to the separation shock-foot unsteadiness, the standard deviation distribution in the intermittent region shows a reduction in maximum rms value, relative to no control, in going from the Ashill device (1\%), to the Anderson configuration (5\%), and finally to the split-Anderson device, which shows $24 \%$ reduction. Beyond the intermittent region, the fluctuation levels in the separated flow region for all test cases remain similar to that for no control. The TRZ configuration, on the other hand, shows a significant rise in the maximum rms value (by $62 \%$ ), followed by RV 1 ( $25 \%$ increase), and finally RV2, which shows a maximum reduction of $26 \%$. For these cases, an increase in fluctuation levels in the separated flow region is indicated.

The surface oil study shows that the variation in the process of flow development from each control device can be related to the variation in location of the CRV convergence (or beginning of oil accumulation line) along the centerline of each device. Both Ashill and Anderson devices show a much earlier formation of convergence location compared to the trapezoidal configuration, for which the line of oil accumulation is relatively delayed. Providing a split in the control device (such as in split-Anderson and ramp-vane configurations) allows the primary air to flow through it that delays or prevents the CRVs formed downstream of such devices to converge early, as was the case with nonsplit devices. The study on ramp-vane devices further shows that the size of the split relative to the device height also seems to be an important parameter, as seen for RV1 $(h / \delta=0.3)$ and $\mathrm{RV} 2(h / \delta=0.5)$ devices. The present study indicates that a smaller intervane spacing of $1.7 h(h / \delta=0.5)$ instead of $3 h(h / \delta=0.3)$ in this case shows a very effective control. From this perspective, 
providing a split size of $1 h$ in the split-Anderson device has also shown favorable results.

\section{Acknowledgments}

The authors wish to thank the Aeronautical Research and Development Board of India for supporting this project. The technical support of Narayana during the model design and fabrication as well as of Perinaygam, Janardhan, and Jones Philip, staff of the $0.3 \mathrm{~m}$ wind-tunnel facility at National Aerospace Laboratories (NAL), during the test campaigns is gratefully acknowledged. Special thanks to Gangadhar, Shanmogan, Charan Singh, and Anupam Mantry of the NAL Belur Model Shop for model fabrication.

\section{References}

[1] Dolling, D. S., and Murphy, M. T., "Unsteadiness of the Separation Shock Wave Structure in a Supersonic Compression Ramp Flowfield," AIAA Journal, Vol. 21, No. 12, 1983, pp. 1628-1634.

[2] Humble, R. A., Scarano, F., and van Oudheusden, B. W., "Unsteady Aspects of an Incident Shock Wave/Turbulent Boundary Layer Interaction," Journal of Fluid Mechanics, Vol. 635, Sept. 2009, pp. 4774. doi:10.1017/S0022112009007630

[3] Barter, J. W., and Dolling, D. S., "Reduction of Fluctuating Pressure Loads in Shock/Boundary-Layer Interactions," AIAA Journal, Vol. 33, No. 10, 1995, pp. 1842-1849. doi: $10.2514 / 3.12736$

[4] Delery, J., "Shock Wave/Turbulent Boundary Layer Interaction and Its Control," Progress in Aerospace Sciences, Vol. 22, No. 4, 1985, pp. 209-280. doi:10.1016/0376-0421(85)90001-6

[5] Viswanath, P. R., "Shock-Wave Turbulent Boundary-Layer Interaction and Its Control: A Survey of Recent Developments," Sadhana, Vol. 12, No. 1,1988 , pp. $45-104$. doi:10.1007/BF02745660

[6] Obery, L. J., and Cubbison, R. W., "Effectiveness of Boundary Layer Removal near Throat of Ramp-Type Side Inlet at Free-Stream Mach Number of 2.0," NACA RM-E54I14, 1954.

[7] Wong, W. F., "The Application of Boundary Layer Suction to Suppress Strong Shock-Induced Separation in Supersonic Inlets," 10th Propulsion Conference, AIAA Paper 1974-1063, 1974. doi:10.2514/6.1974-1063

[8] Fukuda, M. K., Hingst, W. R., and Reshotko, E., "Bleed Effects on Shock/Boundary-Layer Interactions in Supersonic Mixed Compression Inlets," Journal of Aircraft, Vol. 14, No. 2, 1977, pp. 151-156. doi:10.2514/3.58756

[9] Babinsky, H., and Ogawa, H., "SBLI Control for Wings and Inlets," Shock Waves, Vol. 18, June 2008, pp. 89-96. doi:10.1007/s00193-008-0149-7

[10] Babinsky, H., Li, Y., and Pitt Ford, C. W., "Micro Ramp Control of Supersonic Oblique Shock-Wave/Boundary-Layer Interactions," AIAA Journal, Vol. 47, No. 3, 2009, pp. 668-675. doi: $10.2514 / 1.38022$

[11] Szwaba, R., and Doerffer, P., "Shock Wave-Boundary Layer Interaction Control by Streamwise Vortices Mechanics of the 21st Century," Proceedings of the 21st ICTAM, Springer-Verlag, Berlin, Aug. 2004.

[12] Souverein, L. J., and Debiève, J.-F., "Effect of Air Jet Vortex Generators on a Shock Wave Boundary Layer Interaction," Experiments in Fluids, Vol. 49, No. 5, 2010, pp. 1053-1064. doi:10.1007/s00348-010-0854-8

[13] Panaras, A. G., and Lu, F. K., "Micro-Vortex Generators for Shock Wave/Boundary Layer Interactions," Progress in Aerospace Sciences, Vol. 74, April 2015, pp. 16-47. doi:10.1016/j.paerosci.2014.12.006

[14] Lin, J. C., "Review of Research on Low-Profile Vortex Generators to Control Boundary-Layer Separation," Progress in Aerospace Sciences, Vol. 38, Nos. 4-5, 2002, pp. 389-420. doi:10.1016/S0376-0421(02)00010-6

[15] Babinsky, H., Makinson, N. J., and Morgan, C. E., "Micro-Vortex Generator Flow Control for Supersonic Engine Inlets," 45th AIAA Aerospace Sciences Meeting and Exhibit, AIAA Paper 2007-0521, Jan. 2007.

[16] McCormick, D. C., "Shock/Boundary-Layer Interaction Control with Vortex Generators and Passive Cavity," AIAA Journal, Vol. 31, No. 1,
1993, pp. 91-96.

doi: $10.2514 / 3.11323$

[17] Ashill, P. R., Fulker, J. L., and Hackett, K. C., "Studies of Flows Induced by Sub Boundary Layer Vortex Generators (SBVGs)," 40th AIAA Aerospace Sciences Meeting \& Exhibit, AIAA Paper 2002-0968, 2002.

[18] Anderson, B. H., Tinapple, J., and Surber, L., "Optimal Control of Shock Wave Turbulent Boundary Layer Interactions Using Micro-Array Actuation," 3rd AIAA Flow Control Conference, AIAA Paper 20063197, June 2006.

[19] Blinde, P. L., Humble, R. A., Oudheusden, B. W., and Scarano, F., "Effects of Micro-Ramps on a Shock Wave/Turbulent Boundary Layer Interaction," Shock Waves, Vol. 19, Dec. 2009, pp. 507-520. doi:10.1007/s00193-009-0231-9

[20] Giepman, R. H. M., Schrijer, F. F. J., and van Oudheusden, B. W., "Flow Control of an Oblique Shock Wave Reflection with Micro-Ramp Vortex Generators: Effects of Location and Size," Physics of Fluids, Vol. 26, No. 6, 2014, Paper 066101. doi:10.1063/1.4881941

[21] Nolan, W. R., and Babinsky, H., "Comparison of Micro-Vortex Generators in Supersonic Flows," 6th AIAA Flow Control Conference, AIAA Paper 2012-2812, June 2012.

[22] Lee, S., "Large Eddy Simulation of Supersonic Boundary Layer Interaction Control Using Micro-Vortex Generators," Ph.D. Dissertation, Univ. of Illinois at Urbana-Champaign, Urbana, IL, 2009.

[23] Titchener, N., and Babinsky, H., "Control of a Shock-Wave/BoundaryLayer Interaction and Subsequent Subsonic Diffuser Using a Combination of Vortex Generators and Bleed," 50th AIAA Aerospace Sciences Meeting, AIAA Paper 2012-0274, Jan. 2012.

[24] Titchener, N., Babinsky, H., and Loth, E., "The Effects of Various Vortex Generator Configurations on a Normal Shock Wave/Boundary Layer Interaction," 51st AIAA Aerospace Sciences Meeting, AIAA Paper 2013-0018, Jan. 2013.

[25] Holden, H., and Babinsky, H., "Effect of Micro Vortex Generators on Separated Normal Shock/Boundary Layer Interactions," Journal of Aircraft, Vol. 44, No. 1, Jan.-Feb. 2007, pp. 170-174. doi: $10.2514 / 1.22770$

[26] Verma, S. B., and Manisankar, C., "Transition Control of Mach to Regular Reflection Induced Interaction Using an Array of Micro Ramp Vane-Type Vortex Generators," Physics of Fluids, Vol. 27, No. 10, 2015, Paper 107102. doi: $10.1063 / 1.4932405$

[27] Lee, S., Loth, E., and Babinsky, H., "Normal Shock Boundary Layer Control with Various Vortex Generator Geometries," Computers \& Fluids, Vol. 49, No. 1, 2011, pp. 233-246. doi:10.1016/j.compfluid.2011.06.003

[28] Rybalko, M., and Loth, E., "Micro-Ramps for External Compression Low-Boom Inlets," 39th AIAA Fluid Dynamics Conference, AIAA Paper 2009-4206, June 2009.

[29] Li, Q., and Liu, C., "Implicit LES for Supersonic Micro-Ramp Vortex Generator: New Discoveries and New Mechanisms," Modelling and Simulation in Engineering, Vol. 2011, 2011, Paper 934982. doi:10.1155/2011/934982

[30] Sun, Z., Schrijer, F. F. J., Scarano, F., and van Oudheusden, B. W., "The Three-Dimensional Flow Organization Past a Micro-Ramp in a Supersonic Boundary Layer," Physics of Fluids, Vol. 24, No. 5, 2012, Paper 055105. doi:10.1063/1.4711372

[31] Verma, S. B., and Hadjadj, A., "Supersonic Flow Control," Shock Waves, Vol. 25, No. 5, 2015, pp. 443-449. doi:10.1007/s00193-015-0587-y

[32] Bruce, P. J. K., and Colliss, S. P., "Review of Research into Shock Control Bumps," Shock Waves, Vol. 25, No. 5, 2015, pp. 451-471. doi:10.1007/s00193-014-0533-4

[33] Greene, B. R., Clemens, N. T., Magari, P., and Micka, D., "Control of Mean Separation in Shock Boundary Layer Interaction Using Pulsed Plasma Jets," Shock Waves, Vol. 25, No. 5, 2015, pp. 495-505. doi:10.1007/s00193-014-0524-5

[34] Piponniau, S., Dussauge, J. P., Debieve, J. F., and Dupont, P., "A Simple Model for Low-Frequency Unsteadiness in Shock-Induced Separation," Journal of Fluid Mechanics, Vol. 629, June 2009, pp. 87-108. doi: $10.1017 / \mathrm{S} 0022112009006417$

[35] Souverein, L., Dupont, P., Debieve, J., van Dussaugen, J., Oudheusden, B., and Scarano, F., "Effect of Interaction Strength on Unsteadiness in Turbulent Shock-Wave Induced Separations," AIAA Journal, Vol. 48, No. 7, 2010, pp. 1480-1493. doi:10.2514/1.J050093

[36] Ganapathisubramani, B., Clemens, N. T., and Dolling, D. S., "LowFrequency Dynamics of Shock-Induced Separation in a Compression Ramp Interaction," Journal of Fluid Mechanics, 
Vol. 636, Oct. 2009, pp. 397-425.

doi:10.1017/S0022112009007952

[37] Van Driest, E. R., "Turbulent Boundary Layer in Compressible Flows," Journal of the Aeronautical Sciences, Vol. 18, No. 3, 1951, pp. 145-160. doi:10.2514/8.1895

[38] O'Donnell, R. M., "Experimental Investigation at a Mach Number of 2.41 of Average Skin-Friction Coefficients and Velocity Profiles for Laminar and Turbulent Boundary Layers and an Assessment of Probe Effects," NACA TN 3122, 1954.

[39] Edney, B., "Anomalous Heat Transfer and Pressure Distributions on Blunt Bodies at Hypersonic Speeds in the Presence of an Impinging Shock," Aeronautical Research Inst. of Sweden Rept. 115, Stockholm, 1968.

[40] Verma, S. B., and Manisankar, C., "Control of Shock Unsteadiness in Shock Boundary-Layer Interaction on a Compression Corner Using Mechanical Vortex Generators," Shock Waves, Vol. 22, No. 4, 2012, pp. 327-339. doi:10.1007/s00193-012-0369-8

[41] Verma, S. B., and Manisankar, C., "Shock-Wave Boundary-Layer Interaction Control on a Compression Ramp Using Steady Micro AirJets," AIAA Journal, Vol. 50, No. 12, 2012, pp. 2753-2764. doi:10.2514/1.J051577
[42] Rybalko, M., Babinsky, H., and Loth, E., "VGs for a Normal SBLI with a Downstream Diffuser," 40th Fluid Dynamics Conference and Exhibit, AIAA Paper 2010-4464, June-July 2010

[43] Dussauge, J. P., Dupont, P., and Debiève, J. F., "Unsteadiness in Shock Wave Boundary Layer Interactions with Separation," Aerospace Science and Technology, Vol. 10, No. 2, March 2006, pp. 85-91. doi:10.1016/j.ast.2005.09.006

[44] Lu, F. K., Li, Q., and Liu, C., "Microvortex Generators in High-Speed Flow," Progress in Aerospace Sciences, Vol. 53, Aug. 2012, pp. 30-45. doi:10.1016/j.paerosci.2012.03.003

[45] Li, Q., and Liu, C., "Declining Angle Effects of the Trailing Edge of a Microramp Vortex Generator," Journal of Aircraft, Vol. 47, No. 6, 2010, pp. 2086-2095. doi:10.2514/1.C000318

[46] Herges, T., Kroeker, E., Elliott, G., and Dutton, C., "Microramp Flow Control of Normal Shock/Boundary-Layer Interactions," AIAA Journal, Vol. 48, No. 11, 2010, pp. 2529-2542. doi: $10.2514 / 1 . J 050313$

B. Ganapathisubramani Associate Editor 